\title{
Self-organization of critical behavior in controlled general queueing models*
}

\author{
Ph. Blanchard \\ Universitaet Bielefeld \\ Fakultaet fuer Physik \\ D-33619 BIELEFELD (Germany) \\ and \\ M.-O. Hongler \\ STI/ Institut de Production et Robotique/ LPM \\ Ecole Polytechnqiue Fédérale de Lausanne \\ CH-1015 LAUSANNE (Switzerland)
}

July 16, 2003

\begin{abstract}
We consider general queueing models of the $(G / G / 1)$ type with service times controlled by the busy period. For feedback control mechanisms driving the system to very high traffic load, it is shown the the busy period probability density exhibits a generic $-\frac{3}{2}$ power law which is a typical mean field behavior of SOC models.
\end{abstract}

\section{Introduction}

The avalanches occurring in extremal models exhibiting self-organized critically (SOC), (the paradigmatic example being the Bak-Sneppen (BS) model) are defined from the value of a global minimal number $f_{\min }(s)$ as a function of time $s$. Then for any value of the auxiliary parameter $f_{0}$, an $f_{0}$ avalanche of size $S$ is defined as a sequence of $S-1$ successive events with $f_{\min }(s)<f_{0}$ confined between two events

*Partially supported by FCT (Portugal) at CCM University of Madeira 
having $f_{\min }(s) \geq f_{0}$. Accordingly an avalanche is a stochastic process in which the numbers $f_{i}<f_{0}$ play the role of active particles that are randomly created or annihilated. Hence, the avalanche ends when there are no particles left in the system. Clearly the time axis is divided into a series of avalanches. In the BS model, it exists a critical value $f_{c}$ for which the creation of particles is marginally balanced by their annihilation and avalanches of all sizes can happen.

Consider now a queueing systems (QS) which consists of random arrival of customers to a server. Each customer requires a random service time and the system is equipped with a waiting room of unlimited capacity. Here also the time axis can be divided by successive cycle times $(\mathrm{CT})$, a CT being the sum of a busy period (BP) (i.e. the time interval separating two successive instants where the server is starving) and an idle period (i.e periods during which the server is starving). In QS, the role of the $f_{0}$ parameter in the extremal models will now be played by the traffic load $\rho \in[0,1] \subset \mathbb{R}$. The traffic load measures the ratio between the arrival and the service rates and the limiting regime, characterized by $\rho \rightarrow 1$, is well known to lead to a diverging population in the waiting room and BP of all sizes can be realized. In a situation where the traffic load $\rho$ is tuned (and approaches unity from below) by the dynamics of the system itself, the basic elements for a SOC model are present. This is the construction that we adopt in the present paper where a class of controlled QS is studied in the heavy traffic (i.e. $\rho \approx 1$ ) regime. The fact that QS's do play a role in SOC models should not come as a surprise. Indeed the dynamics of the general QS is equivalent to a continuous time, generally nonMarkovian, random walk with a reflecting boundary at the origin (i.e. the empty queue state). Accordingly, the dynamics can be described by Master equations an approach already adopted by [de Boer et al. 94] in their study of mean-field behavior of SOC. Recently the role played by QS in the context of SOC has been pointed out in [Tadic et al 00]. In this paper, the authors introduce the discrete time $E / G / \infty$ model (i.e a QS with Bernoulli arrival and general service processes with an infinite number of parallel servers) to describe the dynamics of the avalanches in the sandpile model. Here, we shall consider the fully general class of continuous time $G / G / 1$ QS for which the arrival and service random processes have finite two first moments but are otherwise arbitrary.

\section{Basic Model}

Let us consider a queueing system (QS) formed by customers arriving to a server. The waiting customers are stored in a waiting room with 
a capacity assumed to be unlimited. The time between successive arrivals $t_{a}$ and the service time $t_{s}$ are independent random variables with cumulative distribution (CDF) given respectively by $A(x)$ and $B(x)$, i.e.

$$
\operatorname{Prob}\left\{0 \leq t_{a} \leq x\right\}=A(x) \text { and } \operatorname{Prob}\left\{0 \leq t_{s} \leq x\right\}=B(x) .
$$

We assume that the CDF's $A(x)$ and $B(x)$ admit moments to any orders and write the averages as:

$$
\frac{1}{\lambda}=\int_{0}^{\infty} x d A(x) \text { and } \frac{1}{\mu}=\int_{0}^{\infty} x d B(x), \quad \rho=\frac{\lambda}{\mu},
$$

with $\rho \in[0.1] \subset \mathbb{R}$ being the traffic load parameter.

As usual for QS, we define the busy period (BP) to be the random variable $U$ characterizing the time interval which begins with the arrival of a customer to the idle server and ends when the server next become idle. We will write:

$G(x)=\operatorname{Prob}\{0 \leq U \leq x\} \quad$ and $\quad g(x) d x=\operatorname{Prob}\{x \leq U \leq x+d x\}$

Consider the evolution of the QS during a time horizon $T$. The time interval $T$ can be divided into successive cycle times (CT) $\xi_{k}$ with $k=1,2,3 \ldots, M$ with $M$ such that $\sum_{k=1}^{M} \xi_{k} \leq T$ and $\sum_{k=1}^{M+1} \xi_{k}>T$ with the definitions

$$
\xi_{k}=U_{k}+I_{k},
$$

and $I_{k}$ being the $k$-th idle period starting directly after the end of the $k$-th BP.

Construction of a controlled queueing system (CQS). The QS will now be equipped with a self-regulating mechanism which adapts the service rate $\mu_{k+1}$ offered during the $(k+1)$-th CT by taking into account the length of the $k$-th BP, namely $U_{k}$. The tuning is somehow a "natural" one, that is to say: the shorter the observed length of the $k$-th $B P$, the stronger the server's availability is reduced during the $k+1$-th $C T$. To mathematically incorporate these qualitative features, we shall write:

$\mu_{k}=\min \left\{\mu_{k-1}, \lambda+\phi\left(U_{k-1}\right)\right\}, \quad \mu_{0}=\lambda+\phi(0)>\lambda>0, \quad k=1,2, \ldots M$, 
with $\phi(x)$ being a monotonously decreasing, positive, function such that:

$$
\lim _{x \rightarrow \infty} \phi(x) \rightarrow 0, \quad \phi(0)=\text { const }>0 .
$$

Using Eqs. (2) and (3), the traffic intensity of the QS fufills:

$$
\begin{gathered}
\rho_{k}=\frac{\lambda}{\mu_{k}}=\frac{\lambda}{\min \left\{\mu_{k-1}, \lambda+\phi\left(U_{k-1}\right)\right\}} \leq \\
\leq \rho_{k+1}=\frac{\lambda}{\mu_{k+1}}=\frac{\lambda}{\min \left\{\mu_{k}, \lambda+\phi\left(U_{k}\right)\right\}} \leq 1 .
\end{gathered}
$$

In view of Eqs.(2), (3) and (4), the traffic load $\rho_{k}$ of the CQS remains constant during the $k$-th $\mathrm{CT}$ and $\rho_{k}$ is increasing as $k$ increases. With the choice given in Eq.(2), the QS modifies itself its traffic load and for $T$ long enough, Eq.(3) implies:

$$
\lim _{k \rightarrow \infty} \rho_{k}=1, \quad \text { (almost surely) }
$$

The actualization of the service rate $\mu_{k}$ given by Eq.(2) does play the role as the tuning of the relaxation probability in the self-organized branching process (SOBP) studied by [Zapperi et al. 95]. It is important to emphasize that for the CQS model the critical regime reached when the traffic load $\rho \rightarrow 1$, does not depend on an external tuning but rather it is controlled by the dynamics of the system itself. This is one of the key features governing SOC systems. In the sequel, we shall show that for asymptotic times (i.e. for $k \rightarrow \infty$ ), the BP probability density $g(x)$ of the CQS, exhibits the $\left(-\frac{3}{2}\right)$ critical exponent characterizing the mean-field behavior of SOC as it is discussed in e.g. [Jensen 98], [Flyvbjerg et al 93], [de Boer et al. 94] and [Zapperi et al. 95].

\subsection{The M/GI/1 queue}

First, we focus on the $M / G / 1$ QS for which we have Poisson arrival (i.e. $A(x)=1-\exp \{-\lambda x\}$ ) and a general $\operatorname{CDF} B(x)$ for the service times. It is well known that for $M / G / 1 \mathrm{QS}$, the $\mathrm{BP}$ solves a functional equation (also known as the Takacs equation), ([Gross et al. 98]):

$$
G^{*}(s)=B^{*}\left(s+\lambda-\lambda G^{*}(s)\right),
$$

where the notations are $G^{*}(s)=\int_{0}^{\infty} e^{-s x} d G(x)$ and $B^{*}(s)=\int_{0}^{\infty} e^{-s x} d B(x)$. From Eq.(6), direct calculations yield, [Takagi 91]: 


$$
\begin{gathered}
\langle U\rangle=\frac{1}{\mu(1-\rho)}, \quad\left\langle U^{2}\right\rangle=\frac{b^{(2)}}{(1-\rho)^{3}}, \quad\left\langle U^{3}\right\rangle=\frac{b^{(3)}}{(1-\rho)^{4}}+3 \lambda \frac{\left[b^{(2)}\right]^{2}}{(1-\rho)^{5}}, \\
\left\langle U^{4}\right\rangle=\frac{b^{(4)}}{(1-\rho)^{5}}+10 \lambda \frac{b^{(2)} b^{(3)}}{(1-\rho)^{6}}+15 \lambda^{2} \frac{\left[b^{(2)}\right]^{3}}{(1-\rho)^{7}},
\end{gathered}
$$

where $b^{(m)}, m=2,3, \ldots$ is the $m$-th moment of $B(x)$, (remember that $\left.b^{1}:=\frac{1}{\mu}\right)$. As from Eq.(5), we have that $\lim _{k \rightarrow \infty} \rho_{k} \rightarrow 1$ all moments of $G(x)$ diverge in this limiting traffic regime. This is the typical signature of a SOC behavior. For the Markovian case characterized by $B(x)=1-\exp \{-\mu x\}$, (i.e. the $M / M / 1$ queue), Eq.(6) can be solved in a closed form and we obtain, [Gross et al. 98]:

$$
g(x) d x=\sqrt{\frac{\mu}{\lambda}} \frac{e^{-(\lambda+\mu) x} I_{1}(x 2 \sqrt{\lambda \mu})}{x} d x,
$$

with $I_{1}$ being a Bessel function. Using the asymptotic expansion:

$$
I_{1}(z) \simeq \frac{e^{z}}{\sqrt{2 \pi z}}+O\left(\frac{1}{z}\right) \text { for } z \rightarrow \infty,
$$

Eq.(7) takes the form:

$$
g(x) \simeq \text { const } \frac{e^{-\mu(1-\sqrt{\rho})^{2} x}}{x^{\frac{3}{2}}} .
$$

Hence, for $\rho \rightarrow 1$, we observe that

$$
g(x) \simeq \operatorname{const} x^{-\frac{3}{2}} .
$$

Hence, the probability density $g(x)$ of the BP exhibits the $\left(-\frac{3}{2}\right)$ power law typical for the mean field behavior of SOC, [Jensen 98], [Flyvbjerg et al. 93], [de Boer et al. 94] and [Zapperi et al. 95 ]. Let us now show that this behavior also holds when general QS are considered.

\subsection{The GI/GI/1 queue in the heavy traffic regime}

Let us now consider general distributions $A(x)$ and $B(x)$ with finite two first moments, (i.e. $G I / G I / 1$ QS). For this type of dynamics, It has been established that in the heavy traffic regime (i.e. $\rho \rightarrow 1$ ), the BP probability density $g(x)$ can be written as, see Eq.(3.10) in [Abate et al. 95]:

$$
g(x) d x \simeq \frac{b}{\sqrt{2 \pi d}} \frac{1}{\sqrt{x^{3}}} e^{-\frac{x(1-\rho)^{2}}{2 d}} d x
$$


with:

$$
d=C_{a}^{2}+C_{s}^{2}
$$

and

$$
b=\frac{1}{1-\left(C_{a}^{2}-1\right) h\left(\rho, C_{a}^{2}, C_{s}^{2}\right)} \quad \text { when } \quad C_{a}^{2} \neq 1,
$$

where: $C_{a}^{2}, C_{s}^{2}$ being the square of the coefficient of variation of the distributions $A(x)$ respectively $B(x)$. The functions $h\left(\rho, C_{a}^{2}, C_{s}^{2}\right)$ read as [Abate et al 95]:

$$
\begin{gathered}
h\left(\rho, C_{a}^{2}, C_{s}^{2}\right)=\frac{1+C_{a}^{2}+\rho C_{s}^{2}}{1+\rho\left(C_{s}^{2}-1\right)+\rho^{2}\left(4 C_{a}^{2}+C_{s}^{2}\right)} \quad \text { when } \quad C_{a}^{2} \leq 1, \\
h\left(\rho, C_{a}^{2}, C_{s}^{2}\right)=\frac{4 \rho}{C_{a}^{2}+\rho^{2}\left(4 C_{a}^{2}+C_{s}^{2}\right)} \quad \text { when } \quad C_{a}^{2}>1 .
\end{gathered}
$$

Observe that for the $\rho \rightarrow 1$ limit, Eq.(9) exhibits the $\left(-\frac{3}{2}\right)$ power law showing that the general class of controlled GI/GI/1 models does exhibit the SOC mean-field exponent.

\section{References}

[Abate et al. 95] J. Abate and W. Whitt. "Limits and approximations for the busy-period distribution in single-server queues". Probab. Theor. Eng. and Informational Sc. 9, (1995), 581-602.

[de Boer et al. 95] J. de Boer, B. Derrida, H Flyvbjerg, A. D. Jackson and T. Wettig. "Simple Model of Self-Organized Biological Evolution" Phys. Rev. Lett. 75, (1994), 906-909.

[Flyvbjerg et al. 93 ] H. Flyvbjerg, P. Bak and K. Sneppen. "Meanfield theory for a simple evolution", Phys. Rev. Lett. 71, (1993), 4087-4090.

[Gross et al. 98] D. Gross and C. M. Harris. Fundamentals of queueing theory. J. Wiley (1998), (third edition).

[Jensen 98] H. J. Jensen Sef-Organized Critically. Emergent Complex Behavior in Physical and Biological Systems. Cambridge Lecture Notes in Physics, 1998.

[Tadic et al 00] B. Tadic and V. Priezzhev. "Scaling of avalanche queues in directed dissipative sandpile" Phys. Rew. E 62, (2000), 3266-3275.

[Takagi 91] H. Takagi. Queueing Analysis- A Foundation and Priority Systems. North Holland (1991). See the Volume 1, chapter 1. 
[Zapperi et al. 95 ] S. Zapperi, K.B. Lauritesen and H.E. Stanley. "Self-organized branching processes: A mean-field theory for avalanches. Phys. Rev. Lett. 75, (1995), 4071-4074. 